\title{
Psychogeography in the Age of the Quantified Self - Mental Map modelling with Georeferenced Personal Activity Data
}

\author{
Sebastian Meier, ${ }^{a}$ Katrin Glinkab \\ ${ }^{a}$ Interaction Design Lab, University of Applied Sciences Potsdam, Germany; meier@fh-potsdam.de \\ ${ }^{b}$ Urban Complexity Lab, University of Applied Sciences Potsdam, Germany; glinka@fh-potsdam.de
}

\begin{abstract}
Personal and subjective perceptions of urban space have been a focus of various research projects in the area of cartography, geography, and related fields such as urban planning. This paper illustrates how personal georeferenced activity data can be used in algorithmic modelling of certain aspects of mental maps and customised spatial visualisations. The technical implementation of the algorithm is accompanied by a preliminary study which evaluates the performance of the algorithm. As a linking element between personal perception, interpretation, and depiction of space and the field of cartography and geography, we include perspectives from artistic practice and cultural theory. By developing novel visualisation concepts based on personal data, the paper in part mitigates the challenges presented by user modelling that is, amongst others, used in LBS applications.
\end{abstract}

Keywords: Mental Maps, Location-based Services, Activity Data, Spatial Analysis, Spatial Behaviour

\section{Introduction}

The ways in which we experience and memorise our interactions with the physical world are highly personal and subjective. To a certain extent, mobile and web-based systems already make use of personalised map approaches that account for subjective views and different modes of representation. Nonetheless, most of the tools that we use to navigate and explore geographic space are standardised mapping tools. This paper will briefly highlight the discourses on personal and mental maps with examples from a cartographic perspective as well as informed by cultural theory and artistic practice. Building upon those discourses, we developed algorithmic approaches to aggregate, analyse, and visualise personal activity data. The visual output is constructed as explorative visualisations, more- over, it also acts as a discursive artefact in order to foster discussion and reflection on individual spatial knowledge, perception, and behaviour.

This paper thus revolves around the research question of how we can model spatial behaviour based on personal georeferenced activity data, while a) building on mental map theories about the influence of mode of transport and b) reflecting artistic and political aspects of psychogeography.

\section{Personal (Mental) Map}

Several academic disciplines, ranging from sociology to psychology and philosophy, have explored the subjective perception of the physical world and its social and mental manifestations. At the same time, the embodiment and representation of political and social implications of (urban) space have been a recurring topic in literature, visual art, and philosophy. In the following two sections we will firstly discuss research from cartography and cognitive sciences and secondly take up artistic and theoretic approaches.

\subsection{Academic Perspectives: Cartography and Cognitive Sciences}

A large body of research has been focused on the question of how people construct spatial knowledge and how this knowledge is used to make sense of the physical world, for instance when performing navigational tasks. A dominant image used in this discourse is the mental map. A general hypothesis within these discourses assumes that an aggregation of the entirety of our experiences form and influence our mental representations of space (Kitchin, 1994; Montello, 2002). Tversky extended the metaphoric concept of the mental map to mental collages, as those mental representations are not solely of map-like forms. Instead, they can rather be understood as collages of experiences in our memory which include stimuli such as images, sounds, or odours (Tversky, 1993). More generally, the correlation between map use and its impact on the shaping of mental maps has been a strong research focus, unravelling findings like the interdependency of orientation in maps and mental maps (mental rotation) (Tversky, 1992; Hintzman et al, 1981). Among these influencing factors, the mode of transport has been identified as one that has significant impact on our perception of space and on how we interact with our environment, an assumption that we will explore further below.

Scientists have tried to capture and visualise mental representations of space, for instance by letting people draw or describe maps (Lynch 1960; Vertesi 2008). Research in neuroscience has produced valuable results in visualising neuronal maps that indicate where spatial information is stored in our brain (Maguire et al. 1997).

However, visualising the resulting mental maps themselves has proven difficult. Taking these constraints 
into account, this paper will not try to visualise a whole mental map or mental collage, but instead focus on one of the previously mentioned influencing factors that form our mental maps: the mode of transport.

Within the research area of mental maps and navigational tasks, a specific strand of research is dedicated to socalled landmarks; a construct which was introduced to the science community by Lynch in 1960 (Lynch 1960). Landmarks are physical constructs, such as buildings, that are of certain significance for a user, for instance as visual aids in order to memorise a route (Foo et al, 2005). Researchers have been trying to understand what makes a physical construct a landmark and how people use them when navigating and memorising urban structures. Most research that focuses on understanding the impact of landmarks includes analysing the given physical structures, their visual features, and how a building stands out from its surroundings (e.g. salience approaches by Caduff et al. (Caduff and Timpf 2008)). This approach does not account for the individual and his or her experiences within this space. The concept of landmarks is, like many other cartographic approaches, characterised by the objective to generalise spatial information insofar as it seeks to identify objects that might be of significance for the general public. Nonetheless, the concept of landmarks also comes into play in our approach. However, we use landmarks in a highly personalised algorithmic spatial representation as a canvas for data mapping and visualisation, as will be further described in section 3 .

\subsection{Psychogeography as Political Practice}

Within the context of Post-Marxism and the spatial analysis of culture, political theorist Frederic Jameson elaborates on "cognitive mapping" from a much more political stance. He contextualises the concept in reference to the notion of an "(unrepresentable, imaginary) global social totality that was to have been mapped" (Jameson 1988: 356) and links it to the context of a socialist agenda. When applied to the design space of an actual rendering of a cognitive map, his envisioning of a new space could be translated into a conceptual model for spatial representation. Jameson describes a "suppression of distance [...] and the relentless saturation of any remaining voids and empty places" (Jameson 1988: 351) . The distortion that arises from this suppression of distance can serve as one of the implications for the design of a visual representation of a mental map. At the same time, Jameson points out that "[a]esthetics is something that addresses individual experience rather than some- thing that conceptualizes the real in a more abstract way" (Jameson 1988: 358). Building on this notion, we deliberately distance our visualisation of personal movement data from seemingly objective and cartographic representations and put an emphasis on metamorphic shapes and aesthetic representations. This, as will be shown in section 4 , is done to a degree that explicitly disregards geographic accuracy and submerges into unique formations of a psychogeographical space that is the subjective city.
The term psychogeography was most prominently coined by the French Situationist International in the 1950s and is understood as "the study of the [...] specific effects of the geographical environment, whether consciously organized or not, on the emotions and behavior of individuals" (Debord 1955). With his work, Debord joined the ranks of writers and artists that, before him, had revolved around the flâneur as a metaphoric figure that strolls the (post) modernist city. This figure has since been subject to significant scrutiny and even re-emerged in the guise of the cyberflâneur which opens a point of reference for our model in the age of the quantified self.

Self-tracking cultures have introduced disciplinary monitoring on a seemingly voluntary level into formerly nondisciplinary (private) spaces (Whitson 2015). With the visualisations described below, we return to Debord's depiction of personal experiences within urban space by using data gathered in self-tracking apps. Reflecting on these references, our mental maps should re-introduce the subjective view to a practice which often claims to provide objective results.

The visualisation deliberately applies distortion and metamorphic shapes to a cartographic and geographic canvas. As a result, our visualisation of personal movement data only fully makes sense in reference to the individual experience of urban space.

\subsection{Duality}

The two previous sections introduced a framework for the concept of mental maps which builds on cartography as well as critical theories. Our approach is informed by both of these discourses and aims at introducing a new perspective on representations of personal spatial behaviour data. Thereby, we seek to bridge the gap between the specialised disciplines and practices of cartography and visualisation, and more artistic and experimental approaches. We believe that visualisations can be extended beyond their function as visual output of (quantitative) data in that they can be staged as individual and aesthetic discursive artefacts, as will be illustrated in the next section. These visual representations can help to identify meaning in da- ta as well as critically reflect on the algorithmic mechanics. Experimental techniques open a window for dialogic interpretation that surpasses abstract information visualisation and traditional cartographic representations.

\subsection{Explorative Tools for Personal Spatial Data Analysis}

Recent developments in the context of openly accessible web technologies have led to a democratisation of coding tools and means of production. This development has also brought about a variety of tools for visualising, exploring, and reflecting upon personal data. The majority of these tools and experimental techniques focuses on information visualisation (see overview by Huang et al. 2015) and interfaces for personal photo collections (e.g. Hyunmo \& Shneiderman, 2000; Thudt et al. 2013). A small subset of tools explores visualisations as a means of accessing personal spatio-temporal data. 
We have identified two directions or types of projects within this subset that are important to our approach. Of these two types we describe an exemplary project each in more detail. The first group of projects focuses on moments, similar to many personal photo collection interfaces, for instance the prototype Visits by Thudt et al. (Thudt et al. 2013; Thudt et al. 2016), shown in Figure 1.

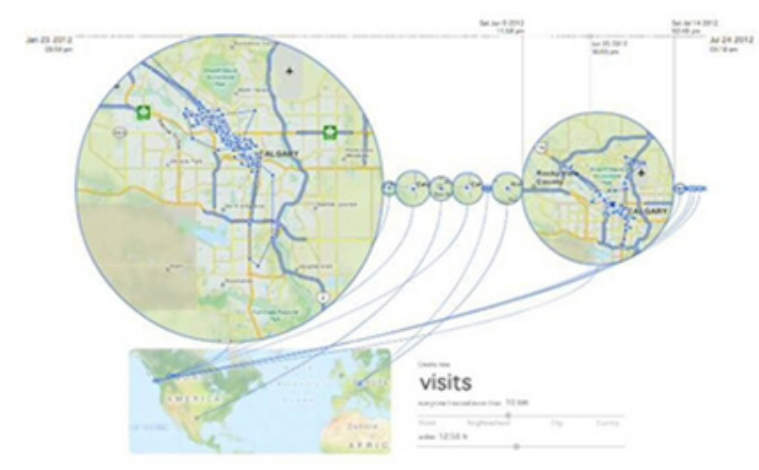

Fig. 1. "Visits showing a location history of six months using a map-timeline approach. This map / timeline hybrid segments the data into stays at places, revealing more information about the temporal aspects of the data." (Thudt et al. 2013:1)

Through the aggregation and clustering of spatiotemporal data, timeline-like visualisations are created that highlight specific time periods. In Visits, those time periods are split into smaller periods based on spatial activity. Next to a contextualising map that helps the reader understand the spatial relationships, the linearity of time dictates the appearance of the main visual elements.

This first group of tools, exemplified by Visits, emanates from specific moments in space and time. The second group of tools also uses data aggregation, but instead of focusing on singular spatio-temporal events, they provide an overview and summaries of the da- ta. This puts them in a direct relationship with life-logging visualisations. A good example for such an application is the Shifted Maps prototype (Otten et al. 2015). Shifted Maps uses activity data to create spatial clusters and visualise the relationships between them in three different dimensions: the position of a location or location cluster; the time it takes to travel between nodes; as well as how frequently the links between locations are used.

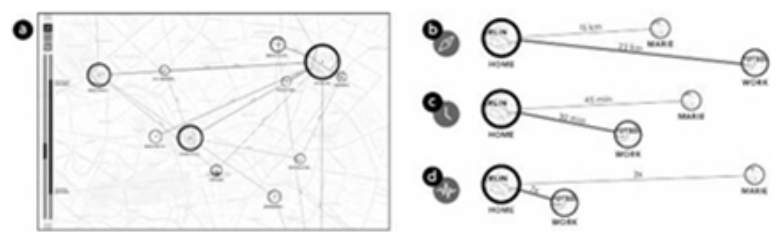

Fig. 2. "The Shifted Maps visualisation (a) and three viewing modes for spatial (b), temporal (c), and frequency (d) networks of places." (Otten et al. 2015:1)

While our approach to some extent bears a similarity to these tools, we are neither interested in summaries nor in a spatial aggregation. Instead, we emphasize the temporal dimension and map the results onto the geographic space, resulting in an overview of spatial behaviour.
The projects described above, as well as our own endeavour strongly build upon principles of time geography. This concept was largely conceptualised in the 1960 s and 70 s by the social geographer Hägerstrand (Hägerstrand, 1970) and investigates social dynamics in a spatio-temporal context. This new wave of projects takes a quantitative, data-driven approach to time geography. Therefore, one might say, that the trajectories we are analysing in the next chapter are digital counterparts to Hägerstrand's "life paths" (Hägerstrand, 1970:10-11).

\section{Algorithmic Approach}

Informed by the theories outlined in section 2, we base our approach on the supposition that mental maps are influenced by many external stimuli, one of them being the way we move through a city (Chorus and Timmermans 2010; Mondschein et al. 2010). Our movements change the way we experience the physical world and thus influence the shaping of mental maps. In our approach we focus on the mode of transport as one such experiential factor, which builds upon work by Mondschein et al., who reported that active or passive navigation influences the quality (richness of detail) of mental maps (Mondschein et al. 2010). Following this supposition, our algorithm tries to calculate the personal level of potential memorisation or spatial knowledge of a certain area. The calculated value is based on trajectory data and the trajectory's mode of transport: from the highest value for walking, to cycling, to the lowest value for motorised transport. The term (potential) memorisation might at first sight seem ambiguous. The term memorisation is often used in contexts of intentional learning, in such, describing how information is moved from the working memory to the long-term memory. In our case, we rather describe the acquisition of knowledge of a certain area. Still, the repetitive interactions and the type of interactions with a certain area help us estimate the quality of spatial knowledge. Therefore, we decided to use the term (spatial) memorisation.

The trajectories are sourced from personal activity data, sometimes referred to as lifelogging data. The term, as it is currently being used, gained attention with the rise of smartphones that were logging data on their users, including GPS trajectories, compass and accelerometer data. Using machine-learning, these datasets are now interpreted and grouped into activities. By calculating speed, rhythm, and movement patterns, algorithms detect the mode of transport and can differentiate between walking, running, cycling, riding a train or travelling on an airplane (Lau 2012; Pennanen \& Kyrölä 2013). While older research on personal activity data was primarily reliant on GPS trajectories (Spek et al, 2009), this new generation of activity data allows more in-depth analysis of individual's activity. Even though latest tracking devices store a richer set of dimensions in activity data, in this paper we will only focus on GPS trajectories combined with the classification of the mode of transport for those trajectories, in order to get insights into one aspect of a person's mental map, the knowledge or rather memorisation of certain spatial areas. To be precise, for 
our prototypical implementation and study we used data gathered through the Moves App (ProtoGeo 2016).

\subsection{Data Aggregation, Cleaning and Organization}

The data collected by the Moves application can be downloaded as JSON files ${ }^{1}$.. The JSON contains the user's trajectories for a specified time period. The Moves application is constantly creating those trajectories based on a GPS signal. Technically, every trajectory consists of a GeoJSON linestring (Butler et al. 2008), a timestamp, and the classification of the mode of transport. Most activity logging devices and applications have to be cautious with their power consumption. Consequently, the precision of the generated georeferenced trajectories is not ideal. One type of error is variation in the data, which means that even though a set of trajectories represent trips on the same road, they will be off by a few meters in any direction. We have factored this in by implementing buffers as described in the next section. The second error are points that are completely off the track. To remove such points, we ran statistics on the whole trajectory set of the individual user and calculated average distances between points for each mode of transport, which allowed us to re- move outliers and replace them with new points between the prior point and the next. Even though the new point will still likely be off track, it gives us a better dataset than with the original outliers.

In addition, the Moves App tries to snap start- and endpoints of trajectories to locations gathered from Foursquare (Foursquare 2014). In cases where no mapped start- and endpoints existed, we used a buffer of 20 meters to generate new locations and mapped them onto the dataset. The 20-meter buffer is needed since points for one location will not be exactly the same due to GPS inaccuracy. This procedure created a cleaned dataset of locations as well as trajectories connecting those locations.

\subsection{Temporal Data Clustering and Network Analysis}

On the basis of the cleaned locations and trajectories, we set out to map the level of assumed spatial knowledge over certain areas by deriving a value for the potential level of memorisation based on the mode of transport. Mondschein et al. (Mondschein et al. 2010) did not develop a formula to calculate these levels, their findings rather indicate an order of memorisation potential for various transport modes. We used their findings and developed a formula that translates visits of a certain part of town by a certain mode of transport into a potential memorisation level that can be used in algorithmic calculations (henceforth referred to as "Potential Memorisation Index", or PMI). This formula serves only as a starting point for our research and has to be refined by more user studies as discussed in section 3.3. Based on

\footnotetext{
${ }^{1}$ Some of the data that can be downloaded from the Moves App will be corrupt. We have included a repair script in the repository mentioned at the end of the paper.
}

the order introduced by Mondschein et al., we used a logarithmic curve that represents an exponential learning curve with a limit. At a certain level of knowledge, each additional spatial interaction will only increase the value of the PMI by a very small amount.

$$
\text { index }=\log (\text { times_visited }+1)^{5.7} * \text { transport_mode }
$$

Fig. 3. Memorisation index, per transport mode (motorised transport $=1$, cycling $=2$ and walking $=3)^{2}$

When combining the PMI from each transport mode, the highest value is chosen. If a user passes a location $x$ times, for example with motorised transport, the formula only generates a very low index.

But if she also walked through the area y times, a higher value is generated. The higher value derived from the walking activity is chosen for further calculations. With these PMIs at hand, we created a 50-meter raster and then intersected the grid cells with the trajectories using a buffer of additional 20 meters to overcome the precision issue (section 3.1), which resulted in a heat-map-like visualisation. Since cities are not made up of 50-meter grid cells, we chose buildings as our final projection canvas and calculated the PMI for each building. The building's geometries are sourced from Open- StreetMap (OpenStreetMap 2016).

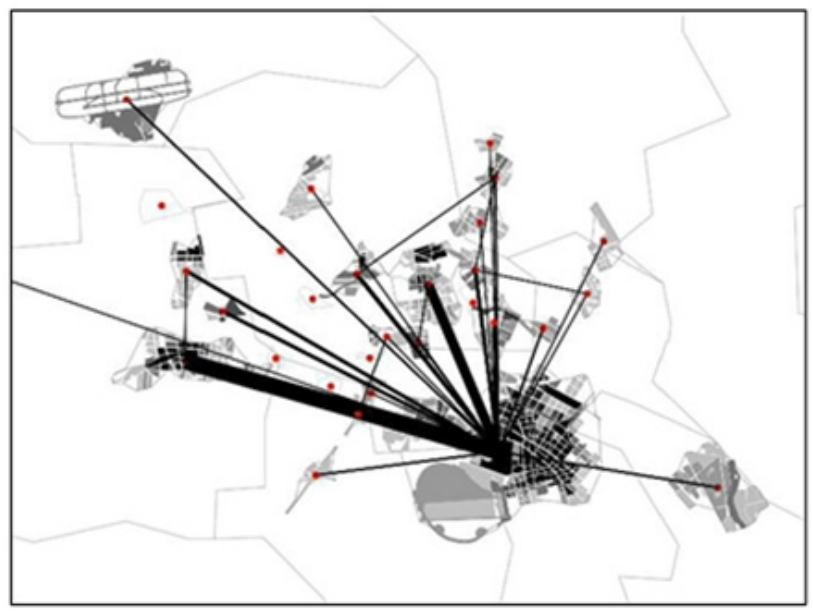

Fig. 4. Trajectories mapped onto buildings. On top of the buildings, the network structure be- tween the walking islands is visualised. Colour-scale: from white unknown to black well known buildings. ${ }^{3}$

Looking at the resulting visualisation (see Fig.4), we identified areas with high PMIs, mainly resulting from walking activities, which we called "walking islands". Those islands were located across the city and connected by other modes of motorised transport (e.g. under-

\footnotetext{
2 Moves successfully classifies running, walking, and cycling. Motorized transport modes depend on the device. Some devices can differentiate between various types, whereas others just return the category transport. Therefore, we use the category transport for all motorised modes.

${ }^{3}$ All exemplary visualisations are generated from the first author's personal activity data from the last 12 month reduced to the extent of the city of Berlin. All visualisations shown in section 3 are webbased, interactive, and can be explored.
} 
ground). To explore those connections of known areas, we created a network graph on top of the islands, highlighting the connections be- tween islands and their respective strength. Strength was calculated through the number of trips, as well as modes of transportation and time spent on trips between islands (Figure 5). This network graph was then translated into a force-directed graph-like concept, allowing us to reorganise the islands depending on their edges. As a result, (see Figure 6), a new city layout emerged that represents the movement and the associated personal experience inspired by the visualisations of the Situationist International.
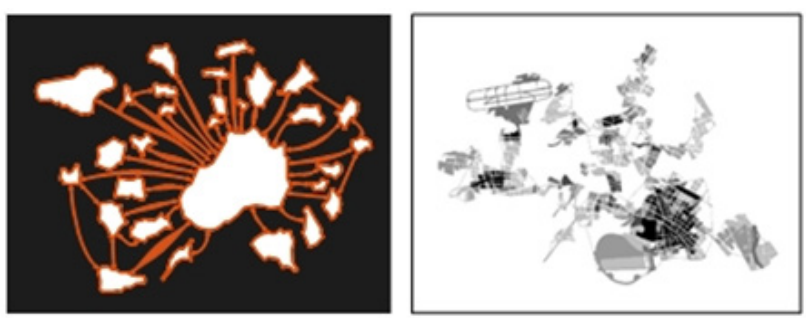

Fig. $5 \& 6$. On the left, the same network represented in a situationist fashion with a manual projection and weighted links (arrows). On the right, using a force directed graph method, the city layout is rearranged and distorted by the suppression of distance.

\subsection{Evaluation of Clusters and Model}

As mentioned in the previous section, several uncertainties become apparent when implementing the concept of Mondschein et al. in an algorithmic approach (apart from the uncertainty of the data as de- scribed in section 3.1). In order to improve our modelling approach, we therefore conducted a preliminary user study with our initial algorithm that incorporates feedback on the assumed level of memorisation.

Recruiting participants for this kind of experiment poses a challenge, as they need to provide us with their personal activity data. Nonetheless, we were able to recruit five participants, three male, two female, between the ages of 25 and 45, all currently living in Berlin.

Patterns of movement through urban space change across time, for example due to switching jobs or moving to a new place. Thus, we segmented the data on the time axis, whereby the dataset for each participant represents only their personal data from last 12 months. This means that for every one of the five participants we used one year of data for the metropolitan region of Berlin / Brandenburg. We also accounted for how long each participant has already been living in Berlin, which was shown to have no impact on the evaluation results.

For the test we selected 22 locations, with a wide range of algorithmically-generated potential memorisation values derived from the mix of transport modes. In addition, we added three locations presumably unknown to the user (at least based on the data we received). We created a web interface in which the user would receive a $360^{\circ}$ image of each location, using Google Street View. The user could change the angle of the image, but not the location. The users were then asked if they knew the location. If so, they were asked to pin-point the location on a map. If the user did not know the location, the correct position was disclosed on the map. If the user recognised the area from the image, or knew the area indicated on the map, they were asked to rank their knowledge on a scale from 1 to 6. From user feedback we learned that in some cases the Google Street View images were quite old and therefore hard to identify. Thus, we allowed people to also rank their knowledge solely based on the position indicated on the map. This applied in cases when they were not able to identify the location based on the image, but later saw on the map that they indeed knew the location.

Our study investigated the performance of our algorithmic approach by comparing the PMI of our algorithm and the reported response from the users. With such a small sample it is difficult to discernibly implicate a correlation or a significant effect, but there is a clear trend in the data, which indicates that our algorithm, even in this untrained phase, performs well. Figure 7 and 8 show that for a high memorisation response our algorithm also calculated a high PMI. Returning to Mondschein et al.'s theory, we see in Figure 9 that are- as with low memorisation responses show less walking and cycling and more motorised transport modes.

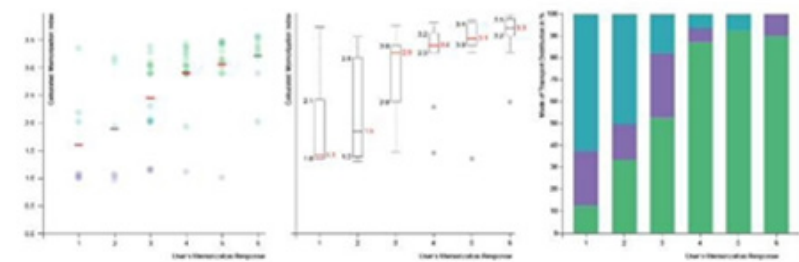

Fig. 7, $8 \&$ 9. The $\mathrm{x}$-axis represents the memorisation response from participants. In fig. 7 and 8 the $y$-axis represents the algorithmically-calculated potential memorisation index. Figure 7 shows the raw data points with averages (red) and fig. 8 an analogous box-plot with medians (red). Figure 9 visualises the percentage for modes of transport for each memorisation response value. Green: walking, purple: cycling, and blue: motorised transport

\section{Reflective Practices}

In addition to the visualisations in Fig. 4-6, we mapped the PMI onto buildings as their height, creating a threedimensional space. As laid out in section 2, our approach is informed by research on mental maps and psychogeography. Drawing on this, we developed a visualisation artefact that deliberately disregards cartographic rules and representational precision. The 3dimensional physical model of the transformed space (see Figure 10) was created by applying rapid prototyping techniques. With this physical model we hope to encourage reflection on the perception of urban structures and the identification of spatial manifestations. The city that served as the backdrop on which the subjective experience of urban space was made and gathered in the form of movement data is now embodied by the distorted proportions and metamorphic shapes that are a dominant feature of the artefact. The artefact should rather trigger personal reflection than analytic exploration. It can be read in the reflection of Jameson's stance on cognitive mapping as the "suppression of distance [...] and the relentless saturation of any remaining voids and empty 
places" (Jameson 1988: 351), while it also addresses the individual experience by means of aesthetic interpretation.
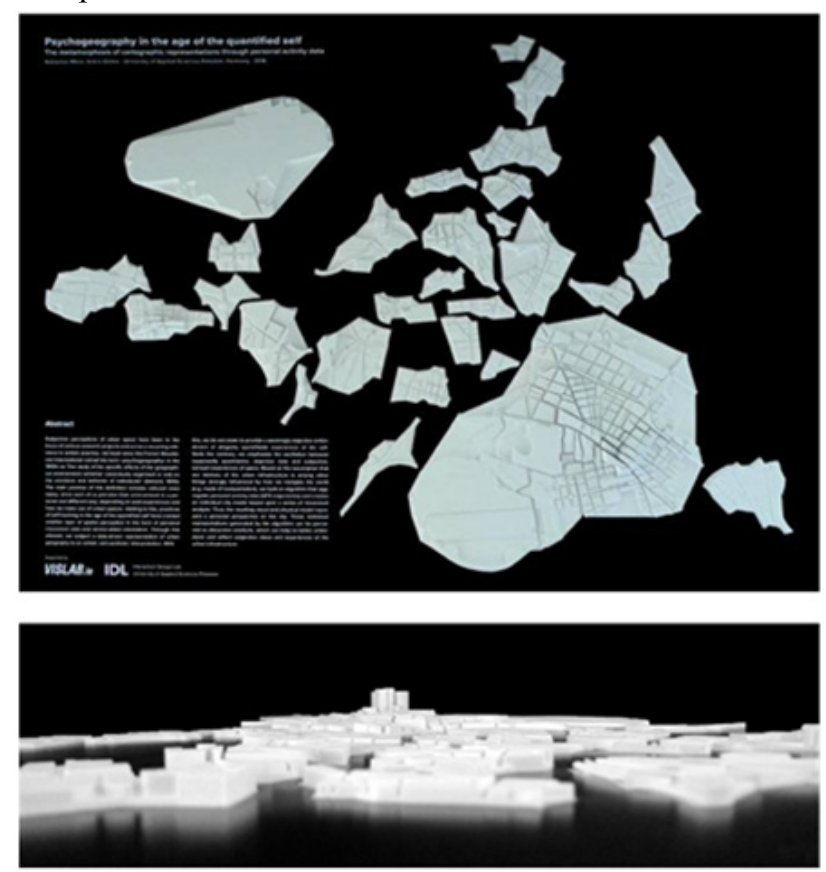

Fig. $10 \& 11$. Images of the 1 meter $x 1$ meter in size physical model, generated from the potential memorisation indices.

\section{Applications \& Future Works}

In our next step of evaluation, we will include a larger sample group to help us improve our algorithm. Furthermore, we want to focus on long-time potential memorisation approaches (forgetting areas, rememorising areas, changes in the (built) environment), to improve the modelling. In addition, we believe our approach, of choosing the mode of transport with the highest resulting PMI, should be reconsidered. As Tversky suggested, our mental maps are derived from all interactions and experiences (Tversky, 1993). Therefore, we should be combining all PMIs from all different modes of transport. When we are able to increase the precision of our method, we see numerous possible applications that go beyond personal reflection. Those applications include improved recommendation methods for location-based services or personalised routing services. Further- more, the comparison of several individual visualisations of personal activity data could also act as a vantage point from which the social and political conditions of accessibility could be traced. By visualising certain aspects of mental maps based on personal activity data we could be able to highlight "projections and repressions in the form of 'go' and 'no-go' space" (Jenks 1995).

From a more data-driven and visualisation-centric perspective we want to refine our algorithms to classify regions by their usage type. Most existing work is using data from a large number of individuals to classify areas. Using only individual data has not been the focus of this work so far. We regard this aspect as especially promising as it would allow us not only to put more emphasis on the personal perspective, but also to focus on privacy and personal data protection.

\section{Conclusion}

Informed by the theoretical perspective of mental maps presented in section 2.1 and 2.2, we provide a data-driven and quantitative perspective of the discourse on mental maps. At the same time, we are aware of the complexities of mental maps and do not try to answer the question of how to visualise mental maps in general. Thus, we have limited our scope to subjective experiences of the urban space on the basis of movement through the city. For this purpose, movement data from self-tracking apps serves as our input phenomena. We presented an algorithm to create a PMI based on mode of transport, inspired by the works of Mondschein et al. The algorithm showed promising results in the preliminary user study.

Although commercial applications like routing services or location recommendation services might also benefit from our algorithm, we above all seek to motivate people to question their own perception of the physical world. Through the visualisations, especially the physical model, we hope to enable new perspectives on personal spatial data and behavioural patterns in urban space.

\section{Acknowledgements}

The code required to run the previously described calculations and to create the visualisations shown in this paper are available on GitHub: github.com/sebastianmeier/MentalMaps.

\section{References}

Butler H, Daly M, Doyle A (2008) GeoJSON Specification. In: geojson.org. http://geojson.org/geojson-spec.html. Accessed 1 Nov 2014

Caduff D, Timpf S (2008) On the assessment of landmark salience for human navigation. Cogn Process 9, pp 249 267.

Chorus C, Timmermans H (2010) Determinants of Stated and Revealed Mental Map Quality: An Empirical Study, Journal of Urban Design 15 (2), pp 211-226

Debord G (1955) Introduction to a Critique of Urban Geography. Les Lèvres Nues \#6, Transla- ted by Ken Knabb.

http://www.cddc.vt.edu/sionline/presitu/geography.html

Foo Patrick, W. Warren, A. Duchon, M. Tarr (2005) Do Humans Integrate Routes Into a Cogni- tive Map? MapVersus Landmark-Based Navigation of Novel Shortcuts. Journal of Experi- mental Psychology, 31 (2), pp 195 215. American Psychology Association

Foursquare (2014) Foursquare for Developers. developer.foursquare.com.

https://developer.foursquare.com/. Accessed 4 Nov 2014

Hägerstrand, Torsten (1970). "What about people in regional science?". Papers of the Regional Science Association. 24 (1), pp 6-21 
Hintzman Douglas L., C. O’Dell, D. Arndt (1981)

Orientation in Cognitive Maps. Cognitive Psychology 13, pp 149-206. Academic Press Inc

D. Huang, M. Tory, B. A. Aseniero, L. Bartram, S. Bateman, S. Carpendale, A. Tang, and R. Woodbury (2015) Personal visualization and personal visual analytics. TVCG, 21(3), pp 420-433. 